\title{
Tentativa de suicídio entre pacientes com uso nocivo de bebidas alcoólicas internados em hospital geral
}

Suicide attempt amongst patients with alcohol misuse admitted

to a general hospital

Daniela Dantas Lima', Renata Cruz Soares de Azevedo', Karla Cristina Gaspar', Viviane Franco da Silva', Marisa Lúcia Fabrício Mauro', Neury José Botega'

\section{RESUMO}

Objetivo: Detectar fatores associados a histórico de tentativa de suicídio (TS) em pacientes internados em hospital geral que fazem uso nocivo de bebidas alcoólicas. Método: 4.352 pacientes admitidos consecutivamente foram avaliados utilizando-se um rastreamento do qual constavam as escalas AUDIT (Alcohol Use Disorder Identification Test) e HAD (Escala Hospitalar de Ansiedade e Depressão). Fixando-se histórico de tentativa de suicídio ao longo da vida como variável dependente, foram realizados testes do qui-quadrado e regressão logística múltipla. Resultados: Uso nocivo de álcool (AUDIT $\geq 8$ ) foi detectado em 423 pacientes. Dentre eles, 60 (14,2\%) apresentavam sintomas de depressão (HAD $\geq 8$ ) e 34 (8\%) tinham histórico de TS. Este se associou a ser adulto jovem [razão de chance $(R C)=3,4$ ], depressão $(R C=6,6)$, uso pregresso de psicofármaco $(R C=7)$ e ter SIDA $(R C=24)$. Conclusão: Os resultados fortalecem a necessidade de detectar e tratar adequadamente condições que, combinadas, aumentam consideravelmente o risco de suicídio.

\begin{abstract}
Objective: To detect factors associated to previous suicide attempt among patients admitted to a general hospital who presented harmful alcohol drinking pattern. Method: 4.352 patients consecutively admitted were screened by means of the AUDIT (Alcohol Use Disorder Identification Test) and HAD (Hospital Anxiety and Depression Scale). Qui-squared tests and multiple logistic regression were performed. Results: 423 individuals presented alcohol harmful use or dependence (AUDIT $\geq 8$ ), 60 (14.2\%) of which had depression (HAD $\geq 8$ ) and 34 (8\%) previous suicide attempt. The latter was more frequent among young adults [odds ratio $(O R)=3.4$ ], those who were depressed $(O R=6.6)$, had previously taken psychotropic medicines $(O R=7)$ and had AIDS $(O R=24)$. Conclusion: Our findings reinforce the need for detection and adequate treatment of conditions that, when toge-

ther, strongly increase the suicide risk.
\end{abstract}

\section{Keywords \\ Alcoholism, suicide attempt, depression, inpatients.}

\section{Palavras-chave \\ Alcoolismo, tentativa de suicídio, depressão, pacientes internados.}

Recebido em

14/3/2010

Aprovado em

30/7/2010 


\section{INTRODUÇÃO}

Suicídio e uso nocivo de álcool encontram-se associados, como demonstram estudos retrospectivos e prospectivos de coorte e pós-morte reunidos em uma revisão'. Indivíduos que abusam ou dependem do álcool têm ideação suicida mais frequentemente e risco mais elevado de tentativas de suicídio, assim como para a consumação do ato em si ${ }^{2,3}$.

O uso nocivo de álcool e a dependência alcoólica associam-se frequentemente a outras doenças psiquiátricas ${ }^{4,5}$. Estudo populacional realizado nos Estados Unidos utilizando uma amostra de 20 mil indivíduos em cinco cidades aponta que, dentre as pessoas com um diagnóstico de transtorno psiquiátrico, um terço tem um segundo transtorno, enquanto, entre os dependentes de álcool, a proporção de comorbidade psiquiátrica atinge $47 \% \%^{6}$. O alcoolismo encontra-se fortemente associado a um histórico de tentativas de suicídio e ambos, a suicídio consumado ${ }^{7,8}$.

O objetivo do presente estudo foi detectar fatores associados a um histórico de tentativas de suicídio ao longo da vida em pacientes que faziam uso nocivo de bebidas alcoólicas e que se encontravam internados em um hospital geral.

\section{MÉTODO}

\section{Delineamento}

Trata-se de um estudo transversal proveniente de dados da fase de rastreamento de um conjunto de estudos controlados e randomizados (estudos de intervenção breve oportuna - EIBO) que avaliaram, entre pacientes internados em um hospital universitário, a eficácia de uma intervenção (entrevista motivacional e contatos telefônicos periódicos, após a alta, ao longo de 12 meses) em casos de depressão, de tabagismo e de uso nocivo de bebidas alcoólicas ${ }^{9-12}$. Entre os pacientes com essa última condição, avaliaram-se variáveis associadas a tentativa de suicídio pregressa.

Este estudo desenvolveu-se no Hospital de Clínicas da Universidade Estadual de Campinas (HC-Unicamp). O HCUnicamp é um hospital terciário com 380 leitos, referência para a rede pública de uma região metropolitana onde vivem 5 milhões de pessoas.

\section{Sujeitos}

Durante o período de recrutamento (outubro de 2007 a outubro de 2008), foram abordados pela equipe de pesquisadores 5.357 (85,3\%) de 6.276 indivíduos com idade maior ou igual a 18 anos que foram admitidos no hospital, excetuando-se as unidades de terapia intensiva e de psiquiatria. Apenas a primeira internação ocorrida no período foi considerada. Cinquenta e nove indivíduos recusaram-se a participar e $946(17,6 \%)$ foram excluídos do estudo.
Os critérios de exclusão foram os seguintes: condições clínicas impossibilitando a entrevista, tais como estado geral grave, distúrbio do nível de consciência e déficit cognitivo $(44,7 \%)$, alta hospitalar prevista dentro de poucas horas (30,1\%), residência fora do estado de São Paulo $(12,7 \%)$ e inexistência de telefone para contatos durante o seguimento (3,5\%). Assim, 4.352 pacientes completaram o questionário de rastreamento.

\section{Instrumentos}

Um instrumento de rastreamento coletou, a partir do prontuário médico, dados sociodemográficos e informações sobre as condições de saúde atual e a razão da internação hospitalar. As seguintes escalas psicométricas foram utilizadas:

Alcohol Use Disorder Identification Test (AUDIT). Compreende 10 questões de múltipla escolha e identifica "risk of hazardous and harmful alcohol use", o que, no presente estudo, foi denominado de uso nocivo de bebidas alcoólicas quando a pontuação (que varia de 0 a 40) foi igual ou maior que 8, segundo validações internacional e nacional ${ }^{13,14}$.

Seção de risco de suicídio do Mini International Neuropsychiatric Interview (MINI-plus, versão 5.0 plus) ${ }^{15,16}$. Em sua seção de risco de suicídio, quatro perguntas questionam sobre comportamento suicida durante o último mês e uma questão, sobre tentativa de suicídio ao longo da vida ("Já fez alguma tentativa de suicídio?"). Essa última foi utilizada no presente estudo para definir histórico de tentativa de suicídio pregressa.

Escala Hospitalar de Ansiedade e Depressão (HAD) ${ }^{17,18}$. Com sete itens de múltipla escolha sobre depressão, foi desenvolvida para ser aplicada em pacientes não psiquiátricos com morbidades clínicas. A determinação dos "casos" de depressão foi estabelecida por pontuação igual ou maior que 8 na subescala de depressão, segundo estudo prévio que validou a HAD, em pacientes internados na mesma instituição ${ }^{18}$.

O tabagismo foi abordado por meio de uma única questão: "Você fumou diariamente durante o último mês?". A intensidade da dor foi avaliada por meio de valores indicados pelo paciente, em uma escala de pontuação de 0 a 10, correspondentes às intensidades no dia da realização da entrevista e nos sete dias precedentes.

Vinte estudantes de medicina foram treinados para o instrumento de rastreamento. $O$ treinamento consistiu de três reuniões de duas horas de duração e compreendeu: introdução ao protocolo da pesquisa, simulações de entrevistas e duas entrevistas com pacientes sob supervisão de médicos psiquiatras. A entrevista de rastreamento era realizada nos dias úteis, à beira do leito, entre 24 e 72 horas após a internação. Levava de 10 a 20 minutos para ser completada.

\section{Análise de dados}

Foram selecionados pacientes com pontuação $\geq 8$ no AUDIT (considerados como tendo feito uso nocivo de be- 
bidas alcoólicas) e divididos em dois grupos, de acordo com a resposta sobre tentativa prévia de suicídio (variável dependente). Para verificar associação ou comparar proporções, foi utilizado o teste qui-quadrado, ou teste exato de Fisher, quando necessário. Para identificar fatores que discriminam o comportamento suicida, foi utilizada a análise de regressão logística univariada e múltipla. O processo de seleção de variáveis aplicado foi o stepwise. Razões de chance com intervalos de confiança de $95 \%$ foram calculadas. O nível de significância adotado para os testes estatísticos foi de 5\%. Utilizou-se o Statistical Analysis System (SAS) versão 8.02.

Este estudo foi aprovado pela Comissão de Ética em Pesquisa da FCM-Unicamp. Cada participante foi informado sobre o estudo e aqueles que concordaram em participar assinaram o termo de consentimento. Nenhum tipo de incentivo financeiro foi oferecido aos participantes.

\section{RESULTADOS}

Dos 4.352 indivíduos entrevistados, 423 (9,7\%) faziam uso nocivo de bebidas alcoólicas. A maioria era de sexo masculino $(87,6 \%)$, casada $(55,6 \%)$, estava em atividade profissional (60,3\%), não estudava no momento $(94,8 \%)$ e possuía pelo menos um filho $(69,9 \%)$.

A análise das duas primeiras questões do AUDIT permite afirmar que, dos que bebiam álcool, 27,7\% faziam-no duas a três vezes por semana e 35,5\%, quatro vezes ou mais por semana. Em um dia típico, 74,1\% bebiam cinco doses de álcool ou mais. A maior prevalência de uso nocivo de álcool $(27,1 \%)$ foi encontrada em pacientes admitidos por causas externas (das quais $85 \%$ deram-se em decorrência de acidentes de trânsito).

Dentre os pacientes que faziam uso nocivo de bebidas alcoólicas, 34 (8\%) tinham histórico de tentativa de suicídio. Na tabela 1, observa-se que o sexo masculino, a depressão, o uso de psicofármacos e a síndrome da imunodeficiência adquirida (SIDA) associaram-se à tentativa de suicídio pregressa. As mesmas características, com exceção de sexo e com o acréscimo de faixa etária jovem $(<36$ anos, variável dicotomizada), permaneceram ao final da análise de regressão múltipla (Tabela 2).

\section{DISCUSSÃO}

Este estudo examinou a associação entre uso nocivo de álcool e tentativa de suicídio pregressa em 423 pacientes, a partir de 4.352 internações consecutivas em um hospital geral. As características que se associaram ao histórico de tentativa de suicídio nessa subpopulação foram: apresentar sintomas de depressão, usar psicofármacos e sofrer de SIDA.
Tabela 1. Características dos pacientes com uso nocivo de bebidas alcoólicas com tentativas de suicídio (análise univariada)

\begin{tabular}{|c|c|c|c|c|}
\hline Característica & $n$ & $\begin{array}{l}\text { Com tentativa de } \\
\text { suicídio }(\%)\end{array}$ & $p$ & \\
\hline Total & 423 & 34 & $(8,0)$ & \\
\hline \multicolumn{5}{|l|}{ Faixa etária (anos) } \\
\hline $18-35$ & 154 & 18 & $(11,7)$ & $0,08^{*}$ \\
\hline $36-50$ & 145 & 12 & $(8,3)$ & \\
\hline $51-62$ & 75 & 2 & $(2,7)$ & \\
\hline$>63$ & 46 & 2 & $(4,3)$ & \\
\hline \multicolumn{5}{|l|}{ Sexo } \\
\hline Masculino & 368 & 26 & $(7,1)$ & $0,05^{* *}$ \\
\hline Feminino & 52 & 8 & $(15,4)$ & \\
\hline \multicolumn{5}{|l|}{ Estado civil } \\
\hline Solteiro & 124 & 14 & $(11,3)$ & $0,22^{* *}$ \\
\hline Casado & & 233 & 15 & $(6,4)$ \\
\hline Viúvo & 16 & 0 & 0,0 & \\
\hline Separado & 46 & 5 & $(10,9)$ & \\
\hline \multicolumn{5}{|l|}{ Atividade do paciente } \\
\hline Dolar & 11 & 1 & $(9,1)$ & $0,94^{*}$ \\
\hline Em atividade profissional & 248 & 19 & $(7,7)$ & \\
\hline Sem atividade profissional & 152 & 13 & $(8,6)$ & \\
\hline \multicolumn{5}{|l|}{ Tem parceiro } \\
\hline Sim & 233 & 15 & $(6,4)$ & $0,15^{*}$ \\
\hline Não & 186 & 19 & $(10,2)$ & \\
\hline \multicolumn{5}{|l|}{ Tem filhos } \\
\hline $\operatorname{Sim}$ & 293 & 20 & $(6,8)$ & $0,14^{*}$ \\
\hline Não & 126 & 14 & $(11,1)$ & \\
\hline \multicolumn{5}{|l|}{ Internação clínica ou cirúrgica } \\
\hline Clínica & 137 & 12 & $(8,8)$ & $0,75^{*}$ \\
\hline Cirurgia & 279 & 22 & $(7,9)$ & \\
\hline \multicolumn{5}{|c|}{ Internação por causa externa ${ }^{* * *}$} \\
\hline Sim & 124 & 11 & $(8,9)$ & $0,72^{*}$ \\
\hline Não & 293 & 23 & $(7,8)$ & \\
\hline \multicolumn{5}{|l|}{ Presença de câncer } \\
\hline Sim & 54 & 3 & $(5,6)$ & $0,59 * *$ \\
\hline Não & 363 & 31 & $(8,5)$ & \\
\hline \multicolumn{5}{|l|}{ Paciente com SIDA ${ }^{* * * * *}$} \\
\hline Sim & 7 & 5 & $(71,4)$ & $0,0001^{* *}$ \\
\hline Não & 411 & 29 & $(7,1)$ & \\
\hline \multicolumn{5}{|l|}{ Uso atual de psicofármacos } \\
\hline Sim & 73 & 11 & $(15,1)$ & $0,02^{* *}$ \\
\hline Não & 311 & 21 & $(6,8)$ & \\
\hline \multicolumn{5}{|c|}{ Uso pregresso de psicofármacos } \\
\hline Sim & 57 & 14 & $(24,6)$ & $0,0001^{* *}$ \\
\hline Não & 361 & 19 & $(5,3)$ & \\
\hline \multicolumn{5}{|l|}{ Uso de tabaco } \\
\hline Sim & 179 & 17 & $(9,5)$ & $0,36^{*}$ \\
\hline Não & 241 & 17 & $(7,1)$ & \\
\hline \multicolumn{5}{|l|}{ Dor na última semana } \\
\hline Sim & 301 & 29 & $(9,6)$ & $0,068^{*}$ \\
\hline Não & 118 & 5 & $(4,2)$ & \\
\hline \multicolumn{5}{|l|}{ Dor no dia da entrevista } \\
\hline Sim & 199 & 19 & $(9,5)$ & $0,30^{*}$ \\
\hline Não & 221 & 15 & $(6,8)$ & \\
\hline \multicolumn{5}{|l|}{ Depressão**** } \\
\hline Sim & 60 & 16 & $(26,7)$ & $0,0001^{* *}$ \\
\hline Não & 359 & 17 & $(4,7)$ & \\
\hline
\end{tabular}

* Qui-quadrado.

** Fischer.

*** $85 \%$ dos quais por acidente automobilístico.

**** Pontuação $\geq 8$ na subescala de depressão na escala Hospitalar de Ansiedade e Depressão (HAD). ***** SIDA: síndrome da imunodeficiencia adquirida. 
Tabela 2. Análise de regressão logística multivariada para tentativa de suicídio prévia em pacientes com uso nocivo de bebidas alcoólicas

\begin{tabular}{lllr}
\hline Variável & Razão de chance & $\begin{array}{l}\text { Intervalo de confiança } \\
\text { 95\% da razão de chance }\end{array}$ & $p$ \\
\hline Idade 18-35 vs. $\geq 36$ anos & 3,4 & $1,3-8,3$ & 0,008 \\
Depressão* & 6,6 & $2,7-16,3$ & $<0,001$ \\
Uso pregresso de psicofármaco & 7,0 & $2,7-17,9$ & $<0,001$ \\
Paciente com SIDA** & 24,1 & $3,4-173,1$ & 0,001 \\
\hline
\end{tabular}

* Pontuação $\geq 8$ na subescala de depressão na escala Hospitalar de Ansiedade e Depressão (HAD).

** SIDA: síndrome da imunodeficiência adquirida.

As limitações metodológicas desta investigação, em grande parte, decorrem de sua natureza transversal, da utilização de instrumentos de rastreamento (que propiciaram dados sumários) e da especificidade da população avaliada.

Não foram incluídas no formulário de inquérito questões sobre a época de ocorrência e a duração dos eventos. Por isso, não se obteve uma sequência temporal que permitisse superar o caráter transversal do estudo e determinar o que ocorrera primeiro: se a depressão, o uso nocivo de álcool ou a(s) tentativa(s) de suicídio, nem se essa última deu-se sob vigência de embriaguez. Questões sobre uso concomitante de drogas ilícitas não foram formuladas aos pacientes.

O formulário de rastreamento não foi além de indicar suspeição de casos. Por isso, não se pode diferenciar entre abuso e dependência de álcool. O AUDIT identifica tão somente hazardous and harmful alcohol use, segundo o expressado em seu manual. Por outro lado, ele é considerado excelente entre os instrumentos de rastreamento para uso de bebidas alcoólicas, com bons níveis de consistência interna, sensibilidade e especificidade ${ }^{19}$. Quando utilizado em atenção primária, foi considerado melhor do que o CAGE e o MAST (Michigan Alcoholism Screening Test) ${ }^{20}$.

Em relação aos "casos" de depressão, deve-se considerar que não se fez confirmação diagnóstica. A suspeição foi dada pela subescala de depressão da HAD, cuja validação foi feita, em estudo prévio, contra uma entrevista psiquiátrica padronizada, com pacientes internados na mesma instituição onde se realizou a presente investigação ${ }^{18}$. Ademais, a prevalência de casos de depressão aqui encontrada (14,2\%) encontra-se próxima às de estudos que utilizaram critérios diagnósticos para depressão maior (major depression) e entrevistas psiquiátricas padronizadas ${ }^{21-23}$. Isso pode se dever, pelo menos em parte, ao uso de um instrumento com características psicométricas muito favoráveis, atestadas por validações local e internacionais. Ademais, a HAD foi especialmente desenvolvida para ser utilizada no tipo de população que investigamos - casos de depressão em pessoas que já sofrem de doenças não psiquiátricas ${ }^{17,18,24}$.

A prevalência global de uso nocivo de bebidas alcoólicas em pacientes internados (9,7\%) não se distanciou do observado em estudos de base populacional realizados em grandes cidades nacionais $(12,3 \%)^{25}$ e em Campinas (8,4\%; onde o AUDIT também foi utilizado) ${ }^{26}$, bem como em hospitais gerais brasileiros, utilizando-se o $\mathrm{MINI}(9,2 \%)^{27}$, ou o AUDIT (12,3\%) 10 anos atrás ${ }^{28}$. O uso nocivo de álcool foi mais frequente entre os admitidos por causas externas (principalmente por acidentes de trânsito), o que vai ao encontro de estudos que relacionam o alcoolismo a acidentes e mortes violentas ${ }^{29-31}$.

A amostra do presente estudo merece consideração especial, uma vez que se trata de indivíduos internados por diversas causas (próximo de um terço das quais em decorrência de acidente automobilístico) e que relataram uso nocivo de álcool ao longo dos últimos 12 meses. Em que medida nossa amostra aproximar-se-ia do encontrado na população geral? O perfil sociodemográfico dos alcoolistas não institucionalizados detectados na comunidade, segundo dados de um inquérito populacional, realizado por meio do AUDIT, na mesma cidade, mostra proporções semelhantes em termos de sexo, faixa etária, estado civil, situação ocupacional e relato de uso pregresso de psicofármaco em torno de $13 \%{ }^{26}$. Os indivíduos de nosso estudo, no entanto, bebem mais frequentemente do que os alcoolistas da comunidade $(35,5 \%$ e $22,3 \%$, respectivamente, bebem em quatro ou mais dias da semana) e em maior quantidade: $74,1 \%$ e $64,8 \%$, respectivamente, bebem, num dia típico, cinco ou mais doses de bebida alcoólica ${ }^{32}$.

Entre os pacientes internados que faziam uso nocivo de álcool, a frequência de $8,2 \%$ de casos de tentativa de suicídio pregressa encontra-se acima dos 5,2\% obtidos entre os que negaram tal uso nocivo, segundo publicação prévia que analisou a mesma base de dados ${ }^{9}$. Ambos os percentuais são maiores do que o observado na população geral (2,8\%, IC95\%: 0,09 - 4,6) da cidade de Campinas, no ano de $2003^{33}$. Esclarecemos que análises referentes a ideação suicida e histórico de tentativa de suicídio para toda a população internada - não só de alcoolistas - encontram-se em vias de publicação $0^{9,10}$.

Esses achados confirmam dados já conhecidos da literatura de que tentativas de suicídio ocorrem com maior frequência entre indivíduos que sofrem de transtornos relacionados ao uso de bebida alcoólica. Kessler et al. ${ }^{34}$ afirmam que indivíduos com alcoolismo tendem a tentar suicídio aproximadamente seis vezes mais que a população geral. Em estudo com 298 alcoolistas, Roy et al. ${ }^{35}$ constatam que 19\% ( $n=$ 57) haviam tentado suicídio. Da mesma forma, estudo conduzido com 3.190 dependentes de bebidas alcoólicas que estavam em tratamento revelou que $16,4 \%(n=522)$ deles reportavam histórias de tentativas ${ }^{36}$. É frequente o histórico de várias tentativas de suicídio ${ }^{37}$.

Entretanto, não são comuns estudos que investiguem especificamente tentativas de suicídio em pacientes que possuam ao mesmo tempo algum transtorno relacionado ao uso de álcool e que estejam internados em hospital geral. 
Estudo realizado em serviço emergencial de hospital geral francês avaliou consumo de álcool recente em pacientes que deram entrada por tentativa de suicídio. $\mathrm{O}$ uso de bebida alcoólica estava presente em $40 \%$ dos casos e, destes, 49\% faziam uso nocivo dessa substância ${ }^{38}$. A prevalência de tentativa de suicídio ao longo da vida encontrada por Pektas et al. ${ }^{39}$, em estudo com pacientes dependentes de bebidas alcoólicas internados, foi de 23,6\%; e em estudo multicêntrico, realizado em hospitais gerais, revelou-se que o álcool estava presente em mais da metade $(54,9 \%)$ dos casos de tentativa de suicídio ${ }^{40}$.

A predominância de tentativas de suicídio entre os adultos jovens (menores de 36 anos) que faziam uso nocivo de álcool coincide com achados de estudos de base populacional ${ }^{33,41,42}$. A ausência de diferença entre os sexos, em nossa amostra, ao contrário do observado na população geral, pode ser resultante do tamanho amostral, como também significar que o uso nocivo de álcool, aliado a outras condições concomitantes, entre os quais a doença física, relativize - diminuindo - a influência do sexo sobre o comportamento suicida.

Pacientes com SIDA, em geral, independentemente de fazerem uso nocivo de álcool, tentam mais o suicídio ${ }^{43,44}$. Em nosso estudo, pacientes que faziam uso de álcool e sofriam de SIDA tiveram o maior risco para tentativa de suicídio (razão de chance igual a 24). Em consonância com nossos achados, um estudo demonstrou que quase metade $(44,3 \%)$ dos pacientes portadores do HIV e com dependência de álcool já havia tentado o suicídio ${ }^{45}$. O alcoolismo, somado ao risco de suicídio aumentado em pessoas acometidas por doenças crônicas e estigmatizantes, pode tornar essa população mais vulnerável a tentativas de suicídio.

A coocorrência do uso nocivo de bebidas alcoólicas e a frequência aumentada de tentativas de suicídio associam-se a outras comorbidades psiquiátricas, hipótese reforçada pela frequência de uso pregresso de psicofármaco e de sintomas de depressão (razões de chance em torno de 7, para cada condição). Depressão e alcoolismo comumente se associam ${ }^{46}$, e o risco de suicídio em pacientes deprimidos com histórico de dependência alcoólica é 59\% mais alto do que em pacientes deprimidos sem histórico de dependência de álcool ${ }^{47}$.

Há, na população estudada, algumas condições - depressão, uso nocivo de álcool, doenças físicas, histórico de tentativa de suicídio - que, além de serem, isoladamente, fatores de risco para o suicídio, juntas provavelmente exercem ação sinérgica de elevação do risco de suicídio ${ }^{1,25,7,7,4,41,47}$. Provavelmente, nossos achados são aplicáveis a outras populações de pessoas que fazem uso nocivo de álcool. Especialmente na população que investigamos, acreditamos que a identificação e a correta abordagem desse problema possam reduzir o risco de suicídio em pacientes que, em decorrência da internação hospitalar, poderão estar mais motivados para iniciar um tratamento em saúde mental.

\section{CONCLUSÃO}

Dos pacientes internados em um hospital geral universitário e que fazem uso nocivo de bebidas alcoólicas, 8,1\% já tentaram suicídio. Esses últimos caracterizam-se por serem mais jovens ( $<36$ anos), por sofrerem de SIDA, por apresentarem depressão e pelo uso pregresso de um psicofármaco.

\section{REFERÊNCIAS}

1. Wilcox CB, Conner KC, Caine ED. Association of alcohol and drug use disorders and completed suicide: an empirical review of cohort studies. Drug Alcohol Depend. 2004;76:11-9.

2. Sher I. Alcohol use and suicide rates. Med Hypotheses. 2005;65:1010-2.

3. Maris RW, Berman AL, Silverman MM. Comprehensive textbook of suicidology. New York: The Guilford Press; 2000, $650 \mathrm{p}$.

4. Grothues J, Bischof G, Reinhardt S, Hapke U, Meyer C, John U, et al. Intention to change drinking behaviour in general practice patients with problematic drinking and comorbid depression or anxiety. Alcohol Alcohol. 2005;40(5):394-400.

5. Schuckit MA, Smith TL, Chacko Y. Evaluation of a depression-related model of alcohol problems in 430 probands from the San Diego prospective study. Drug Alcohol Depend. 2006;82(3):194-203.

6. Robins LN, Regier DA. Psychiatric disorders in America: the epidemiologic catchment area study. New York: Library of Congress; 1991, 449 p.

7. Bertolote JM, Vijayakumar L, Ekeberg 0, Lonnqvist J, Schlebush L, Värnik A, et al. Prevenção do suicídio: um manual para profissionais da saúde em atenção primária. Genebra: OMS: 2000.

8. Owens D, Horrocks J, House A. Fatal and non-fatal repetition of self-harm. Systematic review. Br J Psychiatry. 2002;181:193-9.

9. Botega NJ, Mitsuushi GN, Azevedo RCS, Lima DD, Fanger PC, Mauro MLF, et al. Depression, alcohol use disorders and nicotine dependence among patients at a general hospital. Rev Bras Psiquiatr. 2010;32(3):250-6.

10. Botega NJ, Azevedo RCS, Mauro MLF, Mitsuushi GN, Fanger PC, Lima DD, et al. Factors associated with suicide ideation among medically and surgically hospitalized patients. Gen Hosp Psychiatry. 2010;32(4):396-400.

11. Azevedo RCS, Botega NJ, Mauro MLF, Lima DD, Gaspar KC, Silva VF. General hospital admission as an opportunity for smoking cessation strategies: a clinical trial in Brazil. Gen Hosp Psychiatry. 2010 (no prelo)

12. Fanger PC, Azevedo RCS, Mauro MLF, Lima DD, Gaspar KC, Silva VF, et al. Depressão e comportamento suicida em pacientes oncológicos hospitalizados: prevalência e fatores associados. Rev Assoc Med Bras. 2010;56(2):173-8.

13. Babor TF, La Fuente JR, Saunders J, Grant M. AUDIT - The alcohol use disorders identification test: guidelines for use in primary care. WHO/PAH0. 1992;4:1-29.

14. Figlie NB, Pillon SC, Laranjeira RR, Dunn J. AUDIT identifica a necessidade de interconsulta específica para dependentes de álcool no hospital geral? J Bras Psiquiatr. 1997:46:589-93.

15. Sheehan DV, Lecrubier Y, Sheehan KH, Amorim P, Janavs J, Weiller E, et al. The MiniInternational Neuropsychiatric Interview (MINI): the development and validation of a structured diagnostic psychiatric interview for DSM-IV and ICD-10. J Clin Psychiatry. 1998;59(Suppl 20):22-33.

16. Amorim P. Mini International Neuropsychiatric Interview (MINI): validação de entrevista breve para diagnóstico de transtornos mentais. Rev Bras Psiquiatr. 2000;22:106-15.

17. Zigmond AS, Snaith RP. The Hospital Anxiety and Depression Scale. Acta Psychiatr Scand. 1983:67:361-70.

18. Botega NJ, Bio MR, Zomignani MA, Garcia Júnior C, Pereira WAB. Transtornos de humor em enfermaria de clínica médica e validação de escala de medida (HAD) de ansiedade e depressão. Rev Saude Publica. 1995;29:355-63. 
19. Reinert D, Allen JP. The Alcohol Use Disorders Identification Test (AUDIT): a review of recent research. Alcohol Clin Exp Res. 2002;26(2):272-9.

20. Babor TF, Higgins-Biddle JC. Brief intervention for hazardous and harmful drinking: a manual for use in primary care. Geneva: World Health Organization; 2001.

21. House A. Mood disorders in the physically ill - problems of definition and measurement. J Psychosom Res. 1988;32:345-53

22. Hansen MS, Fink P, Frydenberg M, Oxhøj M, Søndergaard L, Munk-Jørgensen P. Mental disorders among internal medical inpatients: prevalence, detection, and treatment status. J Psychosom Res. 2001;50(4):199-204.

23. Gilbody S, Sheldon T, House A. Screening and case-finding instruments for depression: a meta-analysis. CMAJ. 2008;178(8):997-1003.

24. Bjelland I, Dahl AA, Haug TT, Neckelmann D. The validity of the Hospital Anxiety and Depression Scale - an updated literature review. J Psychosom Res. 2002;52:69-77.

25. Centro Brasileiro de Informação sobre Drogas Psicotrópicas (CEBRID). II Levantamento domiciliar sobre 0 uso de drogas psicotrópicas no Brasil: estudo envolvendo as 108 maiores cidades do país: 2005. Brasília: Secretaria Nacional Antidrogas/Universidade Federal de São Paulo; 2006.

26. Barros MBA, Botega NJ, Dalgalarrondo P, Marín-León L, De Oliveira HB. Prevalence of alcohol abuse and associated factors in a population-based study. Rev Saude Publica. 2007:41(4):502-9.

27. Ferreira MH, Colombo ES, Guimarães PS, Soeiro RE, Dalgalarrondo P, Botega NJ. Suicide risk among inpatients at a university general hospital. Rev Bras Psiquiatr. 2007;29(1):51-4.

28. Figlie NB, Pillon SC, Dunn J, Laranjeira R. The frequency of smoking and problem drinking among general hospital inpatients in Brasil - using the AUDIT and Fagerstrom questionnaires. Sao Paulo Med J. 2000;118(5):139-43.

29. Herttua K, Mäkelä P, Martikainen P. Differential trends in alcohol related mortality: a register-based follow-up study in Finland in 1987-2003. Alcohol Alcohol. 2007;42(5):456-64.

30. Reis DA, Figlie NB, Laranjeira R. Prevalence of substance use among trauma patients treated in a Brazilian emergency room. Rev Bras Psiquiatr. 2006;28(3):191-5.

31. Chen L-H, Baker SP, Li G. Drinking history and risk of fatal injury: comparison among specific injury causes. Accid Anal Prev. 2005;37:245-51.

32. Barros MBA, Marín-León L, Oliveira HB, Dalgalarrondo P, Botega NJ. Perfil do consumo de bebidas alcoólicas: diferenças sociais e demográficas no Município de Campinas, Estado de São Paulo, Brasil, 2003. Epidemiol Serv Saude. 2008;17(4):255-70.
33. Botega JN, Barros MBA, Oliveira HB, Dalgalarrondo P, Marín-León L. Suicidal behavior in the community: prevalence and factors associated with suicidal ideation. Rev Bras Psiquiatr. 2005;27(1):45-53.

34. Kessler RC, Borges $G$, Walters EE. Prevalence of and risk factors for lifetime suicide attempts in the National Comorbidity Survey. Arch Gen Psychiatry. 1999;56:617-25.

35. Roy A, Lamparski D, DeJong J, Moore V, Linnoila M. Characteristics of alcoholics who attempted suicide. Am J Psychiatry. 1990;147:761-5

36. Preuss UW, Schuckit MA, Smith TL, Danko GP, Buckman K, Bierut L, et al. Comparison of 3.190 alcohol-dependent individuals with and without suicide attempts. Alcohol Clin Exp Res. 2002;26(4):471-7.

37. Boenisch S, Bramesfeld A, Mergl R, Havers I, Althaus D, Lehfeld H, et al. The role of alcohol use disorder and alcohol consumption in suicide attempts - A secondary analysis of 1921 suicide attempts. Eur Psychiatry. 2010.

38. Lejoyeux M, Huet F, Claudon M, Fichelle A, Casalino E, Lequen V. Characteristics of suicide attempts preceded by alcohol consumption. Arch Suicide Res. 2008;12(1):30-8.

39. Pektas Ö, Mirsal H, Kalyoncu A, Ünsalan N, Beyazyürek M. Alcohol-dependent patients attempting and not attempting suicide: a comparison. Acta Neuropsychiatr. 2004;16:204-11.

40. Hawton K, Bergen H, Casey D, Simkin S, Palmer B, Cooper J, et al. Self-harm in England: a tale of three cities. Multicentre study of self-harm. Soc Psychiatry Psychiatr Epidemiol. 2007;42(7):513-21.

41. Roy A. Distal risk factors for suicidal behavior in alcoholics: replications and new findings. J Affect Disord. 2003;77:267-71.

42. Diehl A, Laranjeira R. Suicide attempts and substance use in an emergency room sample. J Bras Psiquiatr. 2009;58(2):86-91.

43. Cooperman NA, Simoni JM. Suicidal ideation and attempted suicide among women living with HIV/AIDS. J Behav Med. 2005;28(2):149-56.

44. Gielen AC, McDonnell KA, $\mathrm{O}^{\prime}$ Campo PJ, Burke JG. Suicide risk and mental health indicators: do they differ by abuse and HIV status? Womens Health Issues. 2005;15:89-95.

45. Roy A. Characteristics of HIV patients who attempt suicide. Acta Psychiatr Scand 2003:107(1):41-4.

46. Kaplan HI, Sadock BJ. Kaplan \& Sadock's synopsis of psychiatry: behavioural sciences, clinical psychiatry. 8. ed. Philadelphia: Lippincott Willians \& Wilkins; 1998, 1478 p.

47. Cornelius JR, Salloum IM, Mezzich J, Cornelius MD, Fabrega Jr H, Ehler JG, et al. Disproportionate suicidality in patients with comorbid major depression and alcoholism. Am J Psychiatry. 1995;152(3):358-64. 